\title{
SOME REMARKS ABOUT A THEOREM OF HARTOGS
}

\author{
HENRY B. LAUFER ${ }^{1}$
}

It is a theorem of Hartogs, [4, p. 231 and p. 239] and [2, Theorem 5 , p. 660], that if a bounded domain $G \subset C^{n}, n \geqq 2$, has a connected boundary, then any holomorphic function defined on a connected neighborhood of the boundary of $G$ has a unique holomorphic extension to all of $G$. In this paper, we derive a similar theorem (Theorem 1) for a larger class of sets, the "holomorphic deficiencies" (see Definition 2) introduced by the author in [5, Definition 4.1]. Also, using a theorem of Rossi [7, Theorem 6.6, p. 464], we can show that any compact set $K$ in a connected normal Stein space $S$ of dimension $\geqq 2$ such that $S-K$ is connected, is a removable singularity (Theorem 3 ).

Other new results concern various properties of holomorphic deficiencies and envelopes of holomorphy. Perhaps the most interesting is Theorem 5: if $D$ is an open subset of $M$, a Stein manifold, and $X=M-D$ is a holomorphic deficiency, then $M$ is the envelope of holomorphy of $D$.

Cartan and Schwartz have shown that $H_{*}{ }^{1}(M, \mathcal{O}) \approx 0$, where $M$ is a Stein manifold of dimension at least 2 and $H_{*}{ }^{1}(M, \mathcal{O})$ is the first cohomology with compact supports of $M$ with coefficients in $\mathcal{O}$, the sheaf of germs of holomorphic functions [8, Theorem 4, p. 63]. Using this result, we shall give a direct proof of Theorem 3 for manifolds. See $[8$, p. 66] for another, closely related consequence of Cartan and Schwartz' result.

The author would like to thank Robert C. Gunning for his help and encouragement in writing this article.

Our notation is that of [3]. [6] develops the theory of envelopes of holomorphy for Riemann domains over Stein manifolds. There is a brief summary of needed theorems about such domains in [5].

Definition 1. Let $X$ and $Y$ be point sets in $M$ and $N$ respectively, where $M$ and $N$ are complex manifolds. $X$ and $Y$ are holomorphically equivalent if they have open neighborhoods $X^{\prime}$ and $Y^{\prime}$ in $M$ and $N$ such that there is a biholomorphic map $r: X^{\prime} \rightarrow Y^{\prime}$ which maps $X$ on to $Y$.

Definition 2. A point set $X$ is a complex manifold is a holomorphic deficiency if it is holomorphically equivalent to a set $Y$ such that

Received by the editors March 10, 1966.

1 This paper was written while the author was a National Science Foundation Graduate Fellow. 
$Y=E-D$, where $D$ is a Riemann domain over a Stein manifold and $D$ is an open subset of its envelope of holomorphy $E$.

THEOREM 1. Let $X$ be a holomorphic deficiency in a complex manifold $M$. Let $V$ be an open neighborhood of $X$. Then any holomorphic function defined on $V-X$ has a unique holomorphic extension to all of $V$.

Proof. By our definition of holomorphic deficiency, there are an open neighborhood $X^{\prime}$ of $X$ and a biholomorphic map $r: X^{\prime} \rightarrow Y^{\prime}$ mapping $X$ onto $Y . Y=E-D$, where $E$ is the envelope of holomorphy of $D$. Let $f$ be holomorphic on $V-X$. Without loss of generality, we may restrict $f$ to $\left(V \cap X^{\prime}\right)-X$ and use the biholomorphic map $r$ to consider $f$ defined on a subset $Z$ on the manifold $E . Z=U-Y$, where $U=r\left(V \cap X^{\prime}\right)$ is an open neighborhood of $Y$.

$E=D \cup U$. Cartan's Theorem $\mathrm{B}$ gives $H^{1}(E, \mathcal{O}) \approx 0$ since $E$ is a Stein manifold [6, Theorem 4.6, p. 16]. From the Mayer-Vietoris sequence $[1$, p. 236] we have the following exact sequence

$$
\Gamma(E, \mathcal{\theta}) \rightarrow \Gamma(D, \mathcal{\theta}) \oplus \Gamma(U, \mathcal{\theta}) \rightarrow \Gamma(D \cap U, \mathcal{\theta}) \rightarrow 0 .
$$

However, $U \cap D=U \cap(E-Y)=U-Y=Z$. Thus, given a holomorphic function $f \in \Gamma(Z, \mathcal{O})$ we can write it as the difference of two holomorphic functions. $f=g_{1}+g_{2}, g_{1} \in \Gamma(D, \mathcal{O})$ and $g_{2} \in \Gamma(U, \mathcal{O})$. By hypothesis, $g_{1}$ has a holomorphic extension, also denoted by $g_{1}$, to all of $E$. We can define $f \in \Gamma(U, \mathcal{O})$ by $f=g_{1}-g_{2}$.

To prove uniqueness, consider two possible extensions $f_{1}$ and $f_{2}$. $f_{1}-f_{2}=0$ on $Z$ and so $f_{1}-f_{2}$ provides a holomorphic extension of the zero function from $D$ to all of $E$. This latter extension is unique since $E$ is connected. Hence $f_{1}-f_{2} \equiv 0$ and $f_{1} \equiv f_{2}$.

We see from Theorem 1, that in order to prove that a set is a removable singularity, it suffices to show that it is a holomorphic deficiency. Let $X=E-D$ be a holomorphic deficiency with $E$ the envelope of holomorphy of $D$ and let $Y \subset X$ be closed in $E$ and such that $E-Y=D \cup(X-Y)$ is connected. Then $Y$ is a holomorphic deficiency since $E$ is also the envelope of holomorphy of $D \cup(X-Y)$. Thus, roughly speaking, every closed subset of a removable singularity is a removable singularity.

TheOREM 2. Let $K \subset C^{n}, n \geqq 2$, be such that $K$ is compact and $\boldsymbol{C}^{n}-K$ is connected. Let $U$ be an open neighborhood of $K$. If $f$ is a holomorphic function on $U-K$, then $f$ has a unique holomorphic extension to $U$.

Proof. To apply Theorem 1, we must show that $\boldsymbol{C}^{n}$ is the envelope of holomorphy of $\boldsymbol{C}^{n}-K$. Enclose $K$ in a compact polydisc $Y$. By [3, Theorem 5, p. 20], every function in $C^{n}-Y$ extends to a holo- 
morphic function in $\boldsymbol{C}^{n}$, which proves the assertion.

Theorem 3. Let $K$ be a compact set in a normal, connected Stein space $S$ of dimension $\geqq 2$. If $S-K$ is connected, then any holomorphic function defined on a set $Z$ of the form $Z=U-K$, where $U$ is an open neighborhood of $K$, has a unique holomorphic extension to all of $U$.

PROOF. In order to adapt the proof of Theorem 1, we need only know that every holomorphic function on $S-K$ has a unique extension to all of $S$. This is [7, Theorem 6.6, p. 464].

We can recapture the original form of the Hartogs theorem as follows.

Theorem 4. Let $K$ be a compact set in a connected, normal Stein space $S$ of dimension $\geqq 2$. Let $B$, the set of noninterior points of $K$, be connected. Let $f$ be holomorphic on a connected neighborhood $V$ of $B$. Then there is a unique function $F$ holomorphic in $K \cup V$ such that $F=f$ on $V$.

Proof. Let $K^{\prime}=K-V . K^{\prime}$ is compact. $S-K^{\prime}=(S-K) \cup V$ is connected. Let $U=K^{\prime} \cup V=K \cup V$ and apply Theorem 3 .

Theorem 5. If $D$ is an open subset of $M$, a Stein manifold, and $X=M-D$ is a holomorphic deficiency, then $M$ is the envelope of holomorphy of $D$.

Proof. By Theorem 1, with $V=M$, every holomorphic function on $D$ has a unique holomorphic extension to all of $M$, a Stein manifold. Let $E$ be the envelope of holomorphy of $D$. We must identify $M$ with $E$. $E$ is a Riemann domain over $M$ [6]. $E$ cannot have more than one sheet since some function $f$ would then extend to two different values via different paths in $M$, which it does not. Thus we may consider $E \subset M$. Then $E=M$; for otherwise $E$, since it is a Stein manifold, would have holomorphic functions which do not extend to $M$.

Theorem 6. Let $D^{\prime} \subset D$, with $D$ and $D^{\prime}$ open connected sets in a Stein manifold $M$. Let $Y=M-D$. If $D^{\prime} \cup Y$ is a connected Stein manifold, then $E^{\prime}$, the envelope of holomorphy of $D^{\prime}$, is an open subset of $E$, the envelope of holomorphy of $D$, and $E^{\prime}-D^{\prime}=E-D$.

Proof. Let $X=E-D . X$ is a holomorphic deficiency. $E$ is a Riemann domain over $M$. We shall identify $D$ with its image in $E$. Let $\pi: E \rightarrow M$ be the projection map. Since $\pi$ is a local homeomorphism $\pi(\partial X) \subset \partial Y$. Thus, since $D^{\prime} \cup Y$ is a manifold, $D^{\prime} \cup X$ is open in $E$. Theorem 1 applies to prove that every holomorphic function on $D^{\prime}$ extends uniquely to all of $D^{\prime} \cup X$.

It now suffices to show that $D^{\prime} \cup X$ is a Stein manifold, for then we 
may argue as in the proof of Theorem 4 and conclude that $E^{\prime}=D^{\prime} \cup X$. $\pi(X) \subset D^{\prime} \cup Y$ since each holomorphic function on $D^{\prime}$ extends to $X$ and $D^{\prime} \cup Y$, being a Stein manifold, has functions which have no extension. Hence $\pi\left(D^{\prime} \cup X\right) \subset D^{\prime} \cup Y$. We can now show that $D^{\prime} \cup X$ is holomorphically convex. Let $K$ be a compact set in $D^{\prime} \cup X$ and $\hat{K}$ the holomorphically convex hull of $K$ with respect to the global holomorphic functions on $D^{\prime} \cup X$. Since $D \cup X$ is Stein, if $\hat{K}$ is not compact it has a point in $D \cap \partial D^{\prime}$ as a boundary point. But $\pi\left(D^{\prime} \cup X\right)$ $\subset D^{\prime} \cup Y$ and $D^{\prime} \cup Y$ is a Stein manifold and in particular holomorphically convex. Thus $\hat{K}$ cannot have a point in $D \cap \partial D^{\prime}$ as a boundary point. Hence $\hat{K}$ is compact and $D^{\prime} \cup X$ is holomorphically convex.

As we shall see in the concluding Corollary, the following theorem is an extension of [3, Corollary 3, p. 228] for Stein manifolds.

Theorem 7. Let $X$ be a connected holomorphic deficiency and let $B$ be the set of noninterior points of $X$. Then $B$ is connected.

Proof. Let $X=E-D$ represent $X$ as a holomorphic deficiency. Assume that $B$ is not connected. Then $B=B_{1} \cup B_{2}, B_{1} \cap B_{2}=\varnothing$, with $B_{i} \neq \varnothing$ and $B_{i}$ closed in $B$, hence closed in $X$ and hence closed in $E$. Thus, there exist disjoint open sets $U_{1}$ and $U_{2}$, with $U_{1} \supset B_{1}$ and $U_{2} \supset B_{2}$. Let $V_{i}=U_{i}-X$. By Theorem 1 , every function $f$ which is holomorphic on $V_{1} \cup V_{2}$ extends uniquely to a holomorphic function on $V_{1} \cup V_{2} \cup X$. Let $f=1$ on $V_{1}$ and let $f=2$ on $V_{2}$. For $p \in B_{i}$, there is a polydisc neighborhood of $p$ which is included in $U_{i}$. Hence, by the identity theorem, $f$ must extend to be $i$ in a neighborhood of $p$. So $f=i$ in a neighborhood of $B_{i}$. Now, every component of the interior of $X$ must have a boundary which is a nonempty subset of $B$. By the identity theorem, $f$ must be 1 or 2 on each component of the interior of $X$. We have established the existence of a holomorphic, hence continuous, function on $X$ which assumes only the values 1 and 2. Hence $X$ cannot be connected.

The following theorem is an easy consequence of Theorem 3 but, as mentioned above, we wish to give an independent proof. We can also deduce it from $[8$, p. 66] and Theorem 1.

Theorem 8. Let $D$ be a Riemann domain over a Stein manifold of dimension $\geqq 2$ such that $\mathcal{O}_{D}$ separates points. Let $K$ be a compact set in $D$ such that $D-K$ is connected. If $U$ is an open neighborhood of $K$, then every holomorphic function on $U-K$ has a unique holomorphic extension to $U$.

Proof. Since $\mathcal{O}_{D}$ separates points, $D$ may be considered as an open subset of $E$, its envelope of holomorphy [6]. $E$ is a Stein manifold and $E-K$ is connected. Thus we can replace $D$ by $E$. 
$f$ is holomorphic on $U-K$. There is a $C^{\infty}$ function $r$ on $E$, with compact support $C \subset U$, which is identically 1 on a neighborhood $V$ of $K$ with $\bar{V} \subset U$. Extend $r f$ by 0 on $E-U . \xi=\bar{\partial}(r f)$ is a $C^{\infty}$ form of type $(0,1)$ on $E-K$ with compact support. Extend $\xi$ to be 0 on $K$. Since $H_{*}{ }^{1}(E, \mathcal{O}) \approx 0,\left[8\right.$, Theorem 4, p. 63], there is a (unique) $C^{\infty}$ function $\zeta$ with compact support on $E$ such that $\bar{\partial} \zeta=\xi$. $\zeta$ is holomorphic on $V$ and vanishes outside some compact set $C^{\prime} . \bar{\partial}(\zeta-r f)=0$ on $E-K$, so $\zeta-r f$ is holomorphic on $E-K$. But $\zeta-r f$ is zero outside of $C^{\prime} \cup C$. Hence, since $E-K$ is connected, $\zeta \equiv r f$. But $r f=f$ on $V-K$ so that $\zeta$ provides a holomorphic extension for $f$ to all of $V$.

Uniqueness is proved as before. The zero function must have a zero extension since it is the restriction to $U$ of the zero function on $E-K$ and $E$ is connected.

The following two results are known for Stein spaces as well as for Stein manifolds. See [3, Theorem 2, p. 227] and [3, Corollary 3, p. 228].

THEOREM 9. Let $K$ be a compact, holomorphically convex subset of $M$, a connected Stein manifold of dimension $\geqq 2$. Then $M-K$ is connected.

Proof. $M-K$ can have no relatively compact components. If $U$, a component of $M-K$, satisfied $\bar{U}$ compact, then every holomorphic function $f \in \mathcal{O}_{M}$ would assume its maximum modulus on $\bar{U}$ on $\partial U \subset K$. Thus $U$ would be contained in the holomorphically convex hull of $K$, which is $K$. Thus $U=\varnothing$.

The argument in the proof of Theorem 8 now shows that every holomorphic function on $M-K$ has a unique holomorphic extension to all of $M$; to show that $\zeta \equiv r f$ we need just argue separately for each component. If $M-K=U_{1} \cup U_{2}$ were a decomposition of $M-K$ into disjoint open sets, then $f \equiv 1$ on $V_{1}$ and $f \equiv 2$ on $V_{2}$ would extend to a holomorphic function on $M$. Since $M$ is connected, this is impossible. Thus $M-K$ has one component.

COROLLARY. Any connected compact holomorphically convex subset $K$ of a Stein manifold of dimension $\geqq 2$ has a connected boundary.

Proof. By Theorems 9 and $8, K$ is a holomorphic deficiency. By Theorem $7, K$ has a connected boundary.

\section{BIBLIOGRAPHY}

1. A. Andreotti and H. Grauert, Théorèmes de finitude pour la cohomologie des espaces complexes, Bull. Soc. Math. France 90 (1962), 193-259.

2. S. Bochner, A nalytic and meromorphic continuation by means of Green's formula, Ann. of Math. (2) 44 (1943), 652-673. 
3. R. Gunning and H. Rossi, Analytic functions of several complex variables, Prentice-Hall, Englewood Cliffs, N. J., 1965.

4. F. Hartogs, Einige Folgerungen aus der Cauchyschen Integralformel bei Funktionen mehrerer Veränderlichen, S-B. Münchener Akad. 36 (1906), 223-242.

5. H. Laufer, On sheaf cohomology and envelopes of holomorphy, Ann. of Math. 84 (1966), 102-118.

6. H. Rossi, On envelopes of holomorphy, Comm. Pure Appl. Math. 16 (1963), 9-17.

7. - Vector fields on analytic spaces, Ann. of Math. 78 (1963), 455-467.

8. J.-P. Serre, Quelques problèmes globaux relatifs aux variétés de Stein, Colloque sur les fonctions de plusieurs variables, pp. 57-68, tenu à Brussels, 1953, Thone, Liège, 1953.

Princeton University 